\title{
A Robust Optimization Approach for Magnetic \\ Spacecraft Attitude Stabilization
}

\section{Renato Bruni • Fabio Celani}

\section{Communicated by Firdaus E. Udwadia}

Received: date / Accepted: date

\begin{abstract}
Attitude stabilization of spacecraft using magnetorqers can be achieved by a proportional-derivative-like control algorithm. The gains of this algorithm are usually determined by using a trial-and-error approach within the large search space of the possible values of the gains. However, when finding the gains in this manner, only a small portion of the search space is actually explored. We propose here an innovative and systematic approach for finding the gains: they should be those that minimize the settling time of the attitude error. However, the settling time depends also on initial conditions. Consequently, gains that minimize the settling time for specific initial conditions Renato Bruni, corresponding author Dep. of Computer Control and Management Engineering "Antonio Ruberti", Sapienza University of Rome, Roma, Italy. e-mail: bruni@dis.uniroma1.it Fabio Celani School of Aerospace Engineering, Sapienza University of Rome, Roma, Italy. e-mail: fabio.celani@uniroma1.it
\end{abstract}


cannot guarantee the minimum settling time under different initial conditions. Initial conditions are not known in advance. We overcome this obstacle by formulating a min-max problem whose solution provides robust gains, which are gains that minimize the settling time under the worst initial conditions, thus producing good average behavior. An additional difficulty is that the settling time cannot be expressed in analytical form as a function of gains and initial conditions. Hence, our approach uses some derivative-free optimization algorithms as building blocks. These algorithms work without the need to write the objective function analytically: they only need to compute it at a number of points. Results obtained in a case study are very promising.

Keywords Derivative-free Optimization · Spacecraft Attitude Control · Robust Optimization · Min-Max Problems · Magnetic Actuators

Mathematics Subject Classification (2000) 90C26 - 90C90 - 93D15

\section{Introduction}

Spacecraft attitude control is a very important task in astronautics. It can be obtained by adopting several actuation mechanisms. Among them, magnetorquers are widely used for generation of attitude control torques on satellites flying in low Earth orbits. They consist of planar current-driven coils rigidly placed on the spacecraft typically along three orthogonal axes. They operate on the basis of the interaction between the magnetic dipole moment generated by those coils and the Earth's magnetic field: the interaction with the Earth's field generates a torque that attempts to align the magnetic dipole moment 
in the direction of the field. Magnetorquers have the limitation that control torque is constrained to belong to a plane orthogonal to the Earth's magnetic field, hence a different type of actuator often supports magnetorquers to provide full three-axis control (see [1, Chapter 7$]$ ). Lately, attitude stabilization using only magnetorquers has been considered as a feasible option especially for low-cost micro and nano satellites, and for satellites with a failure in the main attitude control system. In such a scenario, many control laws have been designed, and a survey of various approaches can be found in [2]; in particular, Lyapunov-based design has been adopted in [3-5]. The above works propose feedback control laws that require measures of geomagnetic filed, attitude, and possibly also attitude-rate. These works present theoretical proofs that attitude stabilization is achieved if the adopted feedback gains are positive and a scaling gain is positive and sufficiently small. However, they do not give precise guidelines for determining appropriate numerical values for such parameters, and they are usually determined by a trial-and-error search, carried on until a satisfactory performance of the closed-loop system is obtained. Since the feedback parameters may range over extensive intervals, the corresponding search space is numerically large. Such an approach suffers from two main limitations: (a) it is often very time-consuming; (b) it is not systematic. Hence, even if a satisfactory solution is obtained, it is unknown whether better solutions could be obtained by protracting the search, and also the amount of the possible improvement. This is very inconvenient in practice. 
In this work, we propose an innovative systematic approach for determining the feedback gains: they should be computed as those that minimize the settling time of the attitude error. However, the settling time depends not only on the above gains, but also on a number of other parameters representing the initial conditions of the spacecraft. Thus, we first consider a particular set of initial conditions and search for the gains that minimize the settling time, hereinafter called fixed-conditions optimal gains. Subsequently, we observe that such gains do not guarantee the minimum settling time under different initial conditions. Clearly, there exists a large variety of possible initial conditions, and they are not known a priori. To overcome this issue, we propose to compute the values of the gains that minimize the settling time obtained under the worst initial conditions, so as to provide averagely good results. We model this problem as a min-max problem, and we call robust optimal gains the obtained gains. This problem is quite difficult since the solution of the main minimization problem (upper-level) needs the solution of a maximization problem (lower-level) at every evaluation of its objective function. A decomposition is not possible because the worst initial conditions are not defined in general but they, in turn, depend on the adopted gains. Many real-world problems share this structure; for instance engineering design problems, circuit design problems, and in general the cases when one has to determine a value that should be "not too bad" under many different conditions or perspectives. For related work in this area see, e.g., [6,7] and references therein. 
Our problem presents two additional difficulties: 1) settling time cannot be expressed in analytical form as function of gains and initial conditions, hence the specification of an explicit optimization model is prevented; 2) settling time is discontinuous with respect to both gains and initial conditions. Therefore, our approach uses some derivative-free optimization algorithms as building blocks. These algorithms use no first order information on the objective function, nor do they need its analytical expression. They only need to compute the objective function in a number of points by using simulations. In particular, we use a DIRECT-type global optimization algorithm [8] to tackle the upper-level problem in the above described min-max framework. Roughly speaking, this method is a modification of the standard Lipschitzian approach, that eliminates the need to specify a Lipschitz constant by carrying out simultaneous searches using all possible constants from zero to infinity. Convergence to the global minimum of the upper-level problem is guaranteed by the so-called everywhere dense property, which states that DIRECT is able to generate a set of points which, in the limit, becomes dense in the feasible set [8-10]. To tackle the lower-level problem, we use two methods based on two very different strategies: the mentioned DIRECT algorithm and a more locally oriented optimization algorithm called SDBOX. The latter was initially proposed in [11] as a globally convergent algorithm for the minimization of a continuously differentiable function. However, it can be practically used to optimize different types of functions as a good compromise between efficiency and convergence properties (see also [12]). It is a derivative-free algorithm inspired 
by the strategy underlying gradient-based methods: finding a good feasible descent direction for the objective function, and then performing a sufficiently large step along such direction. However, this algorithm does not need information on the first order derivatives because the good feasible descent direction is determined by investigating the local behavior of the objective function along the coordinate directions. To select the starting points, this method allows to incorporate additional available information on the problem structure, which in our case comes from the physics of the problem.

In conclusion, the main contributions of this work are: $i$ ) the definition of a new systematic approach for the determination of the feedback gains for a proportional-derivative (PD)-like control algorithm; $i i$ ) the development of a min-max formulation to find a robust optimal solution to the problem; iii) a comparison of two derivative-free optimization strategies to tackle similar min-max problems. The work is organized as follows: Section 2 defines the spacecraft model and the control algorithm; Section 3 focuses on the optimization of the feedback gains; Section 4 describes the proposed derivative-free procedure; Section 5 reports computational results.

\section{Spacecraft Model and Control Algorithm}

We introduce the following reference frames to describe the attitude dynamics of an Earth-orbiting rigid spacecraft, and to represent the geomagnetic field.

1. Geocentric Inertial Frame $\mathcal{F}_{i}$. A commonly used inertial frame for Earth orbits is the Geocentric Inertial Frame, whose origin is in the Earth's centre, 
its $x_{i}$ axis is the vernal equinox direction, its $z_{i}$ axis coincides with the Earth's axis of rotation and points northward, and its $y_{i}$ axis completes an orthogonal right-handed frame (see [1, Chapter 2.6.1]).

2. Spacecraft body frame $\mathcal{F}_{b}$. The origin of this right-handed orthogonal frame is attached to the spacecraft and coincides with the satellite's centre of mass; its axes are chosen so that the (inertial) pointing objective is aligning $\mathcal{F}_{b}$ with $\mathcal{F}_{i}$.

We use the following notation: symbol $I$ represents the identity matrix. For the generic $u=\left[\begin{array}{lll}u_{1} & u_{2} & u_{3}\end{array}\right]^{T} \in \mathbb{R}^{3}, u^{\times}$represents the skew symmetric matrix

$$
u^{\times}=\left[\begin{array}{ccc}
0 & -u_{3} & u_{2} \\
u_{3} & 0 & -u_{1} \\
-u_{2} & u_{1} & 0
\end{array}\right]
$$

so that, for $v=\left[\begin{array}{lll}v_{1} & v_{2} & v_{3}\end{array}\right]^{T} \in \mathbb{R}^{3}$, multiplication $u^{\times} v$ is the cross product $u \times v$.

Since the pointing objective consists in aligning $\mathcal{F}_{b}$ to $\mathcal{F}_{i}$, the focus will be on the relative kinematics and dynamics of the satellite with respect to the inertial frame. Let $q=\left[\begin{array}{llll}q_{1} & q_{2} & q_{3} & q_{4}\end{array}\right]^{\mathrm{T}}=\left[\begin{array}{ll}q_{v}^{\mathrm{T}} & q_{4}\end{array}\right]^{\mathrm{T}}$ with $\|q\|=1$ be the unit quaternion representing rotation of $\mathcal{F}_{b}$ with respect to $\mathcal{F}_{i}$. The corresponding attitude matrix is given by (see [13, Section 5.4])

$$
C(q)=\left(q_{4}^{2}-q_{v}^{\mathrm{T}} q_{v}\right) I+2 q_{v} q_{v}^{\mathrm{T}}-2 q_{4} q_{v}^{\times} .
$$

The relative attitude kinematics is given by

$$
\begin{aligned}
& \dot{q}_{v}=\frac{1}{2}\left(q_{v} \times \omega+q_{4} \omega\right) \\
& \dot{q}_{4}=-\frac{1}{2} q_{v}^{T} \omega
\end{aligned}
$$


where $\omega \in \mathbb{R}^{3}$ is the angular rate of $\mathcal{F}_{b}$ with respect to $\mathcal{F}_{i}$ resolved in $\mathcal{F}_{b}$ (see [13, Section 5.5.3]). The attitude dynamics in body frame can be expressed by

$$
J \dot{\omega}=-\omega^{\times} J \omega+T,
$$

where $J \in \mathbb{R}^{3 \times 3}$ is the spacecraft inertia matrix, and $T$ is the control torque expressed in $\mathcal{F}_{b}$ (see [13, Section 6.4]). The spacecraft is equipped with three magnetic coils aligned with the $\mathcal{F}_{b}$ axes which generate the magnetic attitude control torque

$$
T=m_{\text {coils }} \times B^{b}=-B^{b \times} m_{\text {coils }},
$$

where $m_{\text {coils }} \in \mathbb{R}^{3}$ is the vector of magnetic dipole moments for the three coils, and $B^{b}$ is the geomagnetic field at spacecraft expressed in body frame $\mathcal{F}_{b}$ (see [13, Section 12.17]). Let $B^{i}$ be the geomagnetic field at spacecraft expressed in inertial frame $\mathcal{F}_{i}$. Note that $B^{i}$ varies with time at least because of the spacecraft's motion along the orbit. Then, $B^{b}(q, t)=C(q) B^{i}(t)$, which shows explicitly the dependence of $B^{b}$ on both $q$ and $t$.

The following nonlinear time-varying system is obtained by grouping together equations (3), (4), (5), where $m_{\text {coils }}$ is the control input

$$
\begin{aligned}
\dot{q}_{v} & =\frac{1}{2}\left(q_{v} \times \omega+q_{4} \omega\right) \\
\dot{q}_{4} & =-\frac{1}{2} q_{v}^{T} \omega \\
J \dot{\omega} & =-\omega^{\times} J \omega-B^{b}(q, t)^{\times} m_{\text {coils }} .
\end{aligned}
$$

We now need to characterize the time-dependence of $B^{b}(q, t)$, which corresponds to characterizing the time-dependence of $B^{i}(t)$. By adopting the so 
called dipole model of the geomagnetic field (see [14, Appendix H]), and assuming that the orbit is circular of radius $R$, we obtain:

$$
B^{i}(t)=\frac{\mu_{m}}{R^{3}}\left[3\left(\left(\hat{m}^{i}(t)\right)^{\mathrm{T}} \hat{r}^{i}(t)\right) \hat{r}^{i}(t)-\hat{m}^{i}(t)\right]
$$

In equation (7), $\mu_{m}$ is the total dipole strength, $r^{i}(t)$ is the spacecraft's position vector resolved in $\mathcal{F}_{i}$, and $\hat{r}^{i}(t)$ is the vector of the direction cosines of $r^{i}(t)$. The components of vector $\hat{m}^{i}(t)$ are the direction cosines of the Earth's magnetic dipole expressed in $\mathcal{F}_{i}$, which is set equal to

$$
\hat{m}^{i}(t)=\left[\begin{array}{c}
\sin \left(\theta_{m}\right) \cos \left(\omega_{e} t+\alpha_{0}\right) \\
\sin \left(\theta_{m}\right) \sin \left(\omega_{e} t+\alpha_{0}\right) \\
\cos \left(\theta_{m}\right)
\end{array}\right],
$$

where $\theta_{m}$ is the dipole's coelevation, $\omega_{e}=360.99 \mathrm{deg} /$ day is the Earth's average rotation rate, and $\alpha_{0}$ is the right ascension of the dipole at time $t=0$. We use $\mu_{m}=7.74610^{15} \mathrm{~Wb} \mathrm{~m}$ and $\theta_{m}=170.0^{\circ}$ as reported in [15], and we fix time $t=0$ so that $\alpha_{0}=0$.

To characterize the time dependence of $B^{i}(t)$ in (7), one needs to determine an expression for $r^{i}(t)$ which is the spacecraft's position vector resolved in $\mathcal{F}_{i}$. Define a coordinate system $a_{p}, b_{p}$ in the orbital plane, whose origin is at Earth's centre, and with the $a_{p}$ axis coinciding with the line of nodes. Then, the position of satellite's centre of mass is given by

$$
\begin{aligned}
& a^{p}(t)=R \cos (n t+\psi) \\
& b^{p}(t)=R \sin (n t+\psi),
\end{aligned}
$$

where $n$ is the orbital rate, and $\psi$ is the argument of the spacecraft at time $t=0$. The coordinates of the satellite in inertial frame $\mathcal{F}_{i}$ can be easily ob- 
tained from (9) by using an appropriate rotation matrix, which depends on the orbit's inclination incl and on the value $\Omega$ of the Right Ascension of the Ascending Node (RAAN) (see $\left[1\right.$, Section 2.6.2]). An explicit expression for $B^{i}(t)$ can be obtained by plugging the equations of the latter coordinates into (7). The control objective is driving the spacecraft so that $\mathcal{F}_{b}$ is aligned with $\mathcal{F}_{i}$.

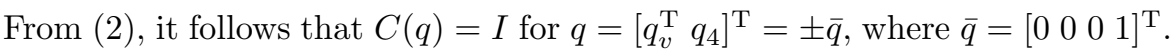
Thus, the objective is designing control strategies for $m_{\text {coils }}$ so that $q_{v} \rightarrow 0$ and $\omega \rightarrow 0$. Reference [5] proposes the following stabilizing proportional-derivative (PD)-like control law, which is a modification of those in [3] and [4]

$$
m_{\text {coils }}=-B^{b}(q, t) \times\left(\epsilon^{2} k_{p} q_{v}+\epsilon k_{d} \omega\right) .
$$

To present the stabilizing properties of the above feedback, we introduce the following matrix

$$
\Gamma^{i}(t)=B^{i}(t)^{T} B^{i}(t) I-B^{i}(t) B^{i}(t)^{T}
$$

along with its average

$$
\Gamma_{a v}^{i}=\lim _{T \rightarrow \infty} \frac{1}{T} \int_{0}^{T} \Gamma^{i}(\tau) d \tau .
$$

Then, the following stabilization result has been obtained in [5], with condition $\Gamma_{a v}^{i}>0$ always fulfilled except for low inclination orbits.

Theorem 2.1 Consider the magnetically actuated spacecraft described by (6). Apply the PD-like control law (10) with $k_{p}>0$ and $k_{d}>0$. If the spacecraft's orbit satisfies $\Gamma_{a v}^{i}>0$, then there exists $\epsilon^{*}>0$ such that for any $0<\epsilon<\epsilon^{*}$, equilibrium $(q, \omega)=(\bar{q}, 0)$ is locally exponentially stable for $(6),(10)$. 


\section{Determination of the Optimal Feedback Gains}

The previous result does not give specific indications on how to choose the feedback gains $k_{p}$ and $k_{d}$, and the scaling factor $\epsilon$. It only states that $k_{p}$ and $k_{d}$ have to be $>0$, and $\epsilon$ has to be $>0$ and smaller than an upper bound $\epsilon^{*}$, whose value is very hard to compute in almost all practical cases. As a matter of fact, in practical implementations, appropriate values for $k_{p}, k_{d}$ and $\epsilon$ are mostly determined by a trial-and-error approach that basically works as follows: a set of significant initial conditions for the spacecraft is considered, some first-guess positive values are chosen for $k_{p}, k_{d}$ and $\epsilon$, and simulations are run. Now, in case the spacecraft's attitude does not converge to the desired one, the value of $\epsilon$ is lowered till convergence is achieved. If the corresponding time behaviors are not satisfactory in terms, for example, of overshoot, speed of convergence, and control inputs' amplitudes, then the values of $k_{p}$ and $k_{d}$ are modified according to the following guidelines used in proportionalderivative control: $k_{p}$ is raised in order to increase the speed of convergence at the expense of larger amplitudes of the control inputs; $k_{d}$ is raised in case the response displays an excessive overshoot. After having modified $k_{p}$ and $k_{d}$, it might be necessary and/or appropriate to assign a different value to $\epsilon$.

The described trial-and-error approach suffers from several limitations. First, this approach is quite time-consuming. Each simulation may require a short time, but running a large number of simulations can often become very demanding. Second, and more important, it is not systematic. Therefore, when satisfactory values of the gains are finally obtained, it is not known whether ex- 
tending the search could lead to new values of the gains that provide an overall better performance of the closed-loop system. Moreover, in case one chooses to extend the search, it is not known how long that should be extended, and not even the amount of the possible improvements that this additional work could produce. In any case, unless performing an exhaustive search for all the possible values of the gains, it can easily happen to neglect values that provide an overall better performance. On the other hand, an exhaustive search is almost always impossible to perform, because the search space is too large to be explored in reasonable time. Indeed, if each gain ranges from 0 to a value of the order of $10^{8}$, which does not appear oversized, then a huge number of pairs of real values should be explored. Theoretically there are infinitely many, and even by limiting the exhaustive search to the precision of integer values, which does not appear excessive, the number of combinations is of the order of $10^{16}$. This implies that, even by running $10^{3}$ simulations per second, the total time would be $10^{13}$ sec., which is about 300,000 years.

Consequently, we propose in this work the following new approach to determine the feedback gains. Since the desired attitude is reached when $q_{v}=0$, we define the settling time $t_{s i}$ for each component $q_{i}$, with $i \in\{1,2,3\}$, as the time needed for $\left|q_{i}\right|$ to become and stay smaller than $\nu$ :

$$
t_{s i}:=\min t \quad \text { s.t. }\left|q_{i}(t)\right| \leq \nu \quad \forall t \geq t_{s i}
$$

Value $0<\nu<1$ depends on how small we wish to keep $q_{i}$. In this work, we set $\nu=0.05$. Then, we define the settling-time $t_{s}$ for the whole quaternion $q$ as the settling time of the slowest component of $q_{v}$, thus $t_{s}:=\max _{i=1,2,3} t_{s i}$. 
Furthermore, rather then expressing feedback (10) by using three parameters $k_{p}, k_{d}$, and $\epsilon$, we rewrite it in terms of only two gains $\kappa_{p}>0$ and $\kappa_{d}>0$ :

$$
m_{\text {coils }}=-B^{b}(q, t) \times\left(\kappa_{p} q_{v}+\kappa_{d} \omega\right) .
$$

Now, if we set the spacecraft's initial conditions to specific values, then we can determine $\kappa_{p}$ and $\kappa_{d}$ so that convergence to the desired attitude is achieved as soon as possible. This can be expressed as the following optimization problem

$$
\min _{\kappa_{p} \geq 0, \kappa_{d} \geq 0} t_{s}
$$

Finally, we observe that the solution to problem (15) would produce extremely large values for $\kappa_{p}$ and $\kappa_{d}$ since, roughly speaking, the larger $\kappa_{p}$ and $\kappa_{d}$, the faster the corresponding closed-loop system. However, too large values of the gains would require the use of magnetic dipole moments probably exceeding the maximum value that can be physically generated by magnetorquers. Hence, we consider the saturated version of feedback (14)

$$
m_{\text {coils }}=-m_{\text {coils }}^{\star} \operatorname{sat}\left(\frac{1}{m_{\text {coils }}^{\star}} B^{b}(q, t) \times\left(\kappa_{p} q_{v}+\kappa_{d} \omega\right)\right),
$$

in which $m_{\text {coils }}^{\star}$ is the saturation limit on each magnetic dipole moment, and sat $: \mathbb{R}^{3} \rightarrow \mathbb{R}^{3}$ is the standard saturation function, defined as follows: given $w \in \mathbb{R}^{3}$, the $i$-th component of $\operatorname{sat}(w)$ is equal to $w_{i}$ if $\left|w_{i}\right| \leq 1$, otherwise it is either 1 or -1 depending on the sign of $w_{i}$. Note that, as stated in [5, Remark 5], local exponential stability of equilibrium $(q, \omega)=(\bar{q}, 0)$ is guaranteed for the closed-loop system even when saturated feedback (16) is employed.

After the definition of the optimization problem (15), we need to solve it numerically. In order to deal with a feasible set that is reasonably bounded and 
not with an infinite search space, we define two upper bounds $\widehat{\kappa_{p}}$ and $\widehat{\kappa_{d}}$ for the gains $\kappa_{p}$ and $\kappa_{d}$. These values can usually be determined for the specific problem, we do this for our case study in Section 5. Thus, the problem becomes as follows, where the dependence of $t_{s}$ on the initial conditions $q_{0}:=q(0)$, $\omega_{0}:=\omega(0)$, and $\psi($ see $(9))$ is explicitly indicated,

$$
\min _{\left(\kappa_{p}, \kappa_{d}\right) \in K} t_{s}\left(\kappa_{p}, \kappa_{d}, q_{0}, \omega_{0}, \psi\right)
$$

and the feasible set is $K=\left\{\left(\kappa_{p}, \kappa_{d}\right): 0 \leq \kappa_{p} \leq \widehat{\kappa_{p}}, 0<\kappa_{d} \leq \widehat{\kappa_{d}}\right\}$. Even though $t_{s}$ obviously depends on $\kappa_{p}$ and $\kappa_{d}$ and on initial conditions, it is practically impossible to express this relationship in analytical form. In addition, $t_{s}$ is discontinuous with respect to both $\kappa_{p}$ and $\kappa_{d}$ and initial conditions. Indeed, it applies for $t_{s}$ the argument presented in [16, p. 233] showing that the settling time of the step response is not continuous with respect to the system's parameters. Because of its intrinsic discontinuity, in optimal control it is often tolerated to replace settling time with more tractable related functions (see [17, Chapter 7]). However, here we keep the settling time as objective, since it best represents the value that we aim to minimize.

Thus, for problem (17), it occurs that: $i$ ) an analytic expression of the objective function is not available, and $i i$ ) the objective function is not continuous with respect to the decision variables. Consequently, optimization approaches that are able to find the minimum of specific classes of functions are not applicable, and derivative-free optimization is needed. 
3.1 From the Simple Min to the Min-Max Problem

Given specific initial conditions $\left(q_{0}, \omega_{0}, \psi\right)$, problem (17) can be solved to optimality by using the global optimization derivative-free technique described in Section 4.1. However, if the initial conditions change, then that solution may be no longer optimal, as shown in the case study in Section 5.1. Since many different initial conditions may occur in practice, a more robust approach would consist in finding the optimal solution to problem (17) under the worst initial condition. Such a worst case optimization is widely used in similar scenarios. The basic idea is paying something, in terms of objective function values, in the easy cases, and obtaining in return advantages in the more difficult situations. However, the worst initial condition is not a priori known, since it depends on the chosen values of $\kappa_{p}$ and $\kappa_{d}$. As a result, the problem cannot be decomposed and should be solved as a whole.

The set of values describing the initial conditions is given by:

$$
\begin{aligned}
S=\left\{\left(q_{0}, \omega_{0}, \psi\right):\right. & \left\|q_{0 v}\right\| \leq 1, q_{04}=\left(1-q_{0 v}^{T} q_{0 v}\right)^{1 / 2}, \\
& \left.\left|\omega_{01}\right| \leq \widehat{\omega_{01}},\left|\omega_{02}\right| \leq \widehat{\omega_{02}},\left|\omega_{03}\right| \leq \widehat{\omega_{03}}, 0 \leq \psi<2 \pi\right\} .
\end{aligned}
$$

Note that $S$ permits any possible initial attitude and any possible initial argument $\psi$ for the spacecraft; it only limits the magnitude of the initial angular rate. Minimizing $t_{s}$ under the worst initial conditions corresponds to the following min-max problem:

$$
\min _{\left(\kappa_{p}, \kappa_{d}\right) \in K} \max _{\left(q_{0}, \omega_{0}, \psi\right) \in S} t_{s}\left(\kappa_{p}, \kappa_{d}, q_{0}, \omega_{0}, \psi\right) .
$$


To apply the technique described in Section 4.1, we need to convert the feasible set of each optimization problem into a hyperrectangle. Since $S$ has not that shape, we express the set $\left\|q_{0 v}\right\| \leq 1$ in spherical coordinates $(\rho, \phi, \theta)$ as follows:

$$
\begin{gathered}
S=\left\{\left(q_{0}, \omega_{0}, \psi\right): q_{01}=\rho \sin \theta \cos \phi, q_{02}=\rho \sin \theta \sin \phi, q_{03}=\rho \cos \theta,\right. \\
q_{40}=\left(1-q_{0 v}^{T} q_{0 v}\right)^{1 / 2}, 0 \leq \rho \leq 1,0 \leq \phi<2 \pi, 0 \leq \theta \leq \pi, \\
\left.\left|\omega_{01}\right| \leq \widehat{\omega_{01}},\left|\omega_{02}\right| \leq \widehat{\omega_{02}},\left|\omega_{03}\right| \leq \widehat{\omega_{03}}, 0 \leq \psi<2 \pi\right\} .
\end{gathered}
$$

The dependence of $t_{s}$ on $q_{0}$ can now be expressed as dependence on the variables $(\rho, \phi, \theta)$; consequently, after having introduced the hyperrectangle

$$
\begin{aligned}
H=\left\{\left(\rho, \phi, \theta, \omega_{0}, \psi\right): 0 \leq \rho \leq 1,0 \leq \phi<2 \pi, 0 \leq \theta \leq \pi\right. \\
\\
\left.\left|\omega_{01}\right| \leq \widehat{\omega_{01}},\left|\omega_{02}\right| \leq \widehat{\omega_{02}},\left|\omega_{03}\right| \leq \widehat{\omega_{03}}, 0 \leq \psi<2 \pi\right\}
\end{aligned}
$$

the min-max problem (18) can be equivalently reformulated as follows

$$
\min _{\left(\kappa_{p}, \kappa_{d}\right) \in K} \max _{\left(\rho, \phi, \theta, \omega_{0}, \psi\right) \in H} t_{s}\left(\kappa_{p}, \kappa_{d}, \rho, \phi, \theta, \omega_{0}, \psi\right)
$$

\section{The Derivative-Free Optimization Procedure}

We describe in this section the min-max procedure proposed to solve problem (19). We begin with the description of the derivative-free optimization algorithms that constitute its building blocks.

\subsection{The Global Strategy}

A major approach in the optimization of a possibly non-differentiable generic function is the use of Lipschitzian methods. An obstacle in using these methods 
is the need of specifying the Lipschitz constant, which is a bound on the rate of change of the function under optimization. However, by suitably dividing the feasible region into sample intervals (hyperrectangles in our multi-dimensional case), and by selecting them in a suitably order, the search may be conducted without knowing the Lipschitz constant. In more detail, our DIRECT-type algorithm [8] works as follows. The feasible region starts as a single hyperrectangle, that is internally normalized to a unit hyperrectangle. At the generic iteration $k$, the algorithm partitions the hyperrectangles obtained from the previous $(k-1)^{t h}$ iteration to form a collection of smaller hyperrectangles $\mathcal{H}^{(k)}=\left\{H_{1}, \ldots, H_{k}\right\}$, and evaluates the objective function at their central points. The choice of the central point is essential for reducing the number of function evaluations. Potentially optimal hyperrectangles within $\mathcal{H}^{(k)}$ are identified, and only these hyperrectangles are further partitioned and investigated in next $(k+1)^{t h}$ iteration of the algorithm. The algorithm stops when the size of the hyperrectangles becomes too small, or when it reaches the maximum number of iterations. This deterministic algorithm will converge to the global optimum of the function if the sampling is dense enough; however the search process may require a large number of function evaluations.

\subsection{The Local Strategy}

A universally known approach to the optimization of sufficiently regular functions is the use of gradient-based methods, which require the computation of the first order derivatives of the objective function. However, when no infor- 
mation on the derivatives is available, one may still try to replicate the basic strategy underlying these methods. Essentially, SDBOX algorithm [11] works as follows. After the selection of a starting point, we cyclically determine a good feasible descent direction for the objective function, and then we perform a sufficiently large step along such direction. The good feasible descent direction is determined by investigating, at each iteration $k$, the local behavior of the objective function at the variation of the $i$-th variable. Variables are selected by simply following a cyclic order. If a move of length $\alpha$ along $d_{i}$ produces a feasible point where the function is sufficiently reduced, then a linesearch technique is performed along $d_{i}$ to provide a suitable stepsize $\alpha_{k}$. Otherwise, the opposite direction $-d_{i}$ is considered, and the same as above is applied. When both $d_{i}$ and $-d_{i}$ do not produce a sufficient decrease, the stepsize $\alpha$ is decreased. The linesearch technique, taken from [11] and derived from [18], does not require information on the slope of the objective function. Even if the convergence of the algorithm was proved in [11] for the case of the minimization of a continuously differentiable function, it has been used to optimize also different types of functions. It is computationally faster than the global strategy; however a poor choice of the initial point leads to poor solutions.

\subsection{The Whole Procedure}

To simplify and generalize the description of the proposed robust optimization approach, that could also be applied to different problems sharing the same structure, we now rename the set of variables $\left(\kappa_{p}, \kappa_{d}\right)$ as $x$ belonging to a 
feasible set $F_{x}=\left\{l b_{i} \leq x_{i} \leq u b_{i}, i=1, \ldots, n\right\} \subset \mathbb{R}^{n}$ (in our case $F_{x}=K$ and $n=2)$, and the set of initial conditions $\left(\rho, \phi, \theta, \omega_{0}, \psi\right)$ as $y$ belonging to a feasible set $F_{y}=\left\{l b_{j} \leq y_{j} \leq u b_{j}, j=1, \ldots, m\right\} \subset \mathbb{R}^{m}$ (in our case $F_{y}=H$ and $m=7$, since $\omega_{0}$ has 3 components). Denote by $g(x, y)$ the function providing the objective value (in our case $t_{s}$ ). Its analytical expression is not available, but its value can be computed by means of a software simulation.

The upper-lever minimization problem $\min _{x \in F_{x}} g(x, y)$ should be solved only once by means of a global strategy, since we have no elements to suppose that the solution is in the vicinity of some pre-determinable starting point. Therefore, we use an external loop that applies the global strategy. This loop computes, up to a maximum number of function evaluations maxeval_ext, the value of $g$ obtained for certain values of $x$. Let $\bar{x}$ be one of them; then, the corresponding evaluation needs the solution of one lower-lever maximization problem $\max _{y \in F_{y}} g(\bar{x}, y)$. Thus, the lower-lever problem should be solved repeatedly up to maxeval_ext times, and reducing its computation time to a few seconds is crucial. In addition to solving it with the global strategy using a necessarily small maximum number of function evaluations maxeval_int (option 1), we also tackle it with the local strategy, allowing the same number of function evaluations (option 2). Moreover, we can further favor the solution of the maximization problem by using any information that happens to be available in the choice of the starting points. In our case, using the physics of the problem, we suppose that good solutions are in the vicinity of extremal values of angular velocity. Hence, we use also the local strategy with multi-start from 
different starting points, still allowing maxeval_int total function evaluations (option 3). The whole procedure is as follows:

\section{Procedure: Solve min-max}

Input: A $g(x, y)$ computable by means of a software simulation for $x_{i} \in$ $\left[l b_{i}, u b_{i}\right]$ with $i=1, \ldots, n$ and $y_{j} \in\left[l b_{j}, u b_{j}\right]$ with $j=1, \ldots, m$

Output: A robust optimum value $x^{*}=\arg \min _{x \in F_{x}}\left(\max _{y \in F_{y}} g(x, y)\right)$

\section{External loop:}

Solve the upper-level problem $\min _{x \in F_{x}} f(x)$, with $f(x)=\max _{y \in F_{y}} g(x, y)$ by using DIRECT for maxeval_ext evaluations of $f$ and return $x^{*}$

The $k$-th evaluation works with point $x^{(k)}$

\section{Internal loop:}

Solve the lower-level problem $\max _{y \in F_{y}} g\left(x^{(k)}, y\right)$

$$
\text { by using: }\left\{\begin{array}{l}
\text { DIRECT performing maxeval_int evaluations of } g \\
\text { SDBOX single start performing maxeval_int evaluations of } g \\
\text { SDBOX multistart with } s \text { starting points, performing } \\
\frac{\text { maxeval_int }}{s} \text { evaluations of } g \text { for each of them }
\end{array}\right.
$$

\section{Results}

We apply our approach to solve the case study presented in [5]. The spacecraft's inertia matrix is $J=\operatorname{diag}[27,17,25] \mathrm{kg} \mathrm{m}^{2}$; the saturation level for each magnetic dipole moment is $m_{\text {coils }}^{*}=10 \mathrm{~A} \mathrm{~m}^{2}$. The inclination of the orbit is 
incl $=87^{\circ}$, and the orbit's altitude is $450 \mathrm{~km}$; the corresponding orbital period is about $5609 \mathrm{~s}$; the value $\Omega$ of RAAN is equal to 0 . From the previous parameters, an upper bound $\widehat{\kappa_{p}}$ for $\kappa_{p}$ can be determined as follows. For the considered orbit, it can be shown by simulation that $\left\|B^{i}(t)\right\| \geq B_{\min }=2.4 \times 10^{-5} \mathrm{~T}$; since $\left\|B^{b}(q, t)\right\|=\left\|B^{i}(t)\right\|$, it follows that $\left\|B^{b}(q, t)\right\| \geq B_{\text {min }}$, as well. Assume that the attitude sensor has a resolution in terms of quaternion's component of $q_{s}=0.04$. Then, an upper bound for $\kappa_{p}$ can be found by enforcing that each component of the term $B^{b}(q, t) \times \kappa_{p} q_{v}$ (see (16)) does not exceed the saturation limit $m_{\text {coils }}^{*}=10 \mathrm{~A} \mathrm{~m}^{2}$ when $B^{b}(q, t)$ and $q_{v}$ are orthogonal, $\left\|B^{b}(q, t)\right\|=B_{\text {min }},\left|q_{i}\right|=q_{r}$ for one index $i \in 1,2,3$, and $q_{i}=0$ for the other ones. This is achieved if $\kappa_{p}$ is smaller than $m_{\text {coils }}^{*} /\left(B_{\min } q_{r}\right)=1.0417 \times 10^{8}$; thus, we set $\widehat{\kappa_{p}}=10^{8}$. Assuming that the attitude rate sensor has a resolution of $\omega_{r}=4 \times 10^{-4} \mathrm{rad} / \mathrm{s}$, by a parallel argument it follows that an upper bound for $\kappa_{d}$ is given by $m_{\text {coils }}^{*} /\left(B_{\min } \omega_{r}\right)=1.0417 \times 10^{9}$; thus, we set $\widehat{\kappa_{d}}=10^{9}$.

\subsection{Fixed Initial Conditions}

Consider an initial state characterized by attitude equal to the target attitude $q_{0}=\bar{q}$ (which corresponds to having $\rho=0$ and any value for $\phi$ and $\theta$ ) and by the following initial angular rate

$$
\omega_{0}=\left[\begin{array}{lll}
0.02 & 0.02 & -0.03
\end{array}\right]^{\mathrm{T}} \mathrm{rad} / \mathrm{s}
$$

This state can occur when the spacecraft possesses the desired attitude with no angular momentum, and an impact with an object (e.g., a piece of debris or 
a tiny meteorite) occurs, leading to an instantaneous change in the spacecraft's angular rate. In addition, we choose for $\psi$ a random value over the interval $[0,2 \pi[$ by setting $\psi=0.332 \mathrm{rad}$.

Gains $\kappa_{p}$ and $\kappa_{d}$ were determined in [5] by trial-and-error and set equal to $\kappa_{p}=2 \times 10^{5}$ and $\kappa_{d}=3 \times 10^{8}$. The corresponding time behaviors are reported in Fig. 1, and the settling time turns out to be $t_{s}=4.002$ orbital
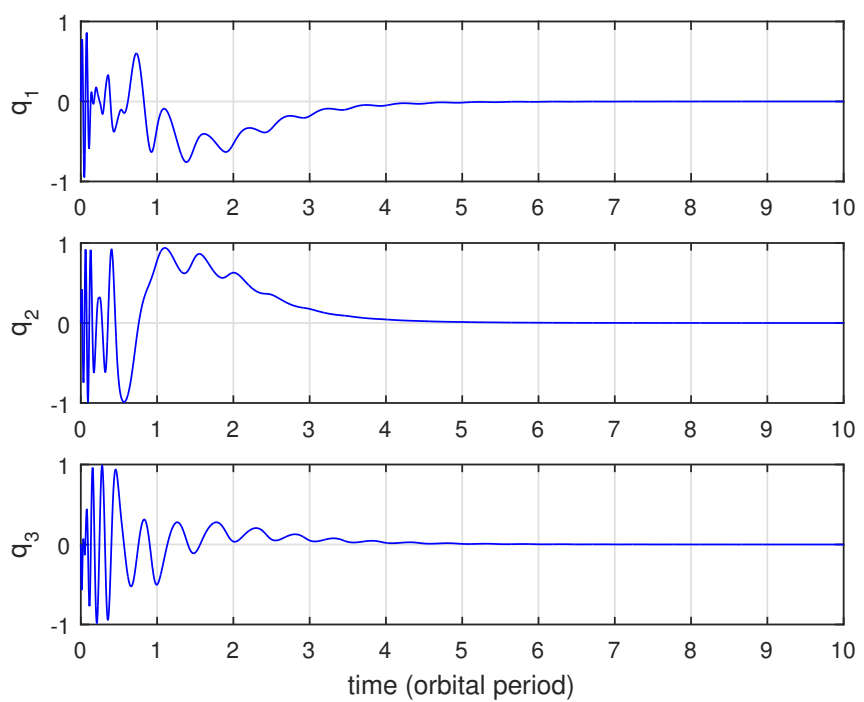

Fig. 1 Evolutions from initial state (20) with feedback gains determined by trial-and-error.

periods. Note that the vast majority of gains' values would result in settling times larger than 10 orbital periods. On the contrary, using the optimization approach described in Section 4.1 with 32,821 iterations (corresponding to 251.5 secs. of computation), we obtain the following fixed-conditions optimal gains:

$$
\kappa_{p}=205761.316872 \quad \kappa_{d}=99382716.049383 .
$$


The corresponding time evolutions are represented in Fig. 2, and the obtained
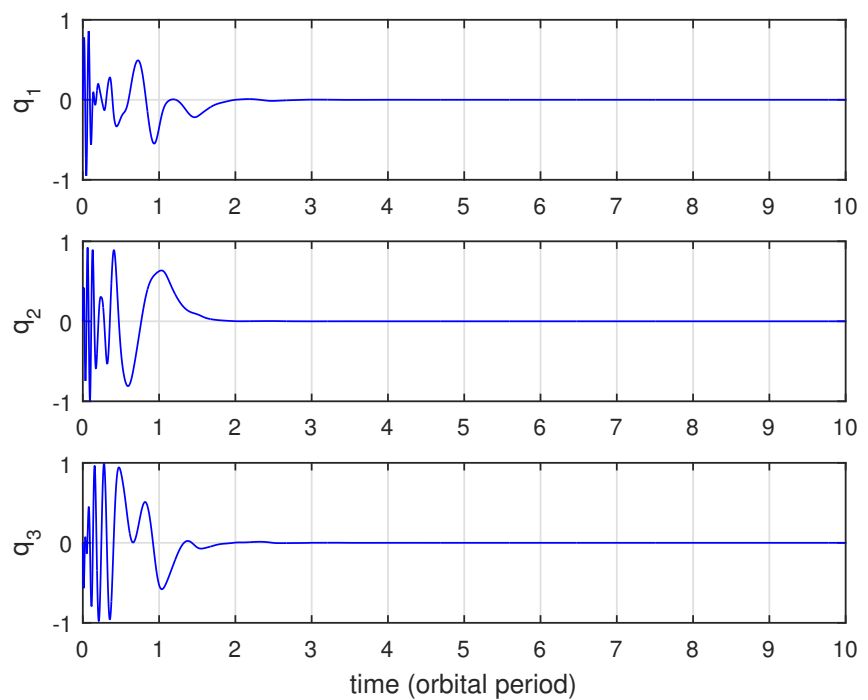

Fig. 2 Evolutions from initial state (20) with fixed-conditions optimal feedback gains (21).

optimal settling time is $t_{s}=1.775$ orbital periods. Thus, with respect to the trial-and-error approach, a significant improvement in convergence speed to the target attitude is achieved. Note that even if the values of the gains determined by trial-and-error are not very far from the optimal ones, the reduction of the settling time is quite large.

However, if we consider different initial conditions, the above gains might be no longer optimal. For example, in the case of initial conditions having the same attitude $q_{0}=\bar{q}$ (i.e., $\rho=0$ and any $\phi$ and $\theta$ ), same argument $\psi=0.332$ rad, but different initial angular rate

$$
\omega_{0}=\left[\begin{array}{lll}
0.1 & 0.1 & 0.1
\end{array}\right]^{\mathrm{T}} \mathrm{rad} / \mathrm{s},
$$


the above gains yield a settling time $t_{s}=3.562$, and they are no longer optimal, as shown in Section 5.2. The corresponding time evolutions are in Fig. 3.
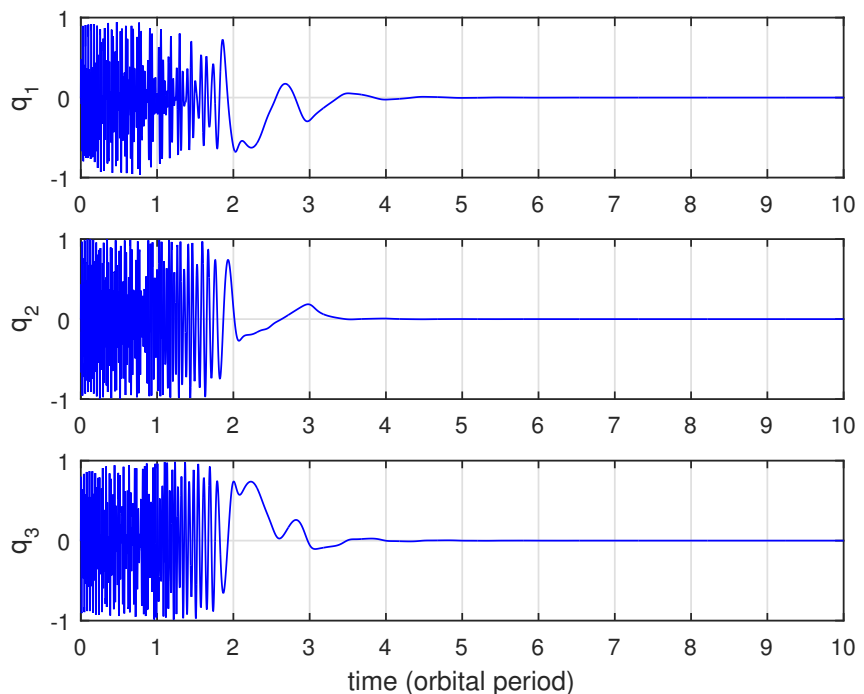

Fig. 3 Evolutions from initial state (22) with feedback gains (21).

Indeed, optimal gains would need to be recomputed for every new initial condition. If we consider the practical variability of the initial conditions, it is clearly impossible to do so. As explained in Section 3.1, a valid solution consists in finding the values of the gains that provide the best value under the worst initial condition. The worst initial condition corresponding to gains (21) is

$$
\begin{gathered}
\rho=0.5 \quad \phi=0.0 \quad \theta=2.356194490192345 \quad \psi=1.570796326794897 \\
\omega_{0}=\left[\begin{array}{lll}
-0.1 & 0.1 & 0.1
\end{array}\right]^{\mathrm{T}} .
\end{gathered}
$$

Under condition (23), the above values of $\kappa_{p}$ and $\kappa_{d}$ yield a settling time $t_{s}=4.338$. The corresponding time evolutions are represented in Fig. 4. 

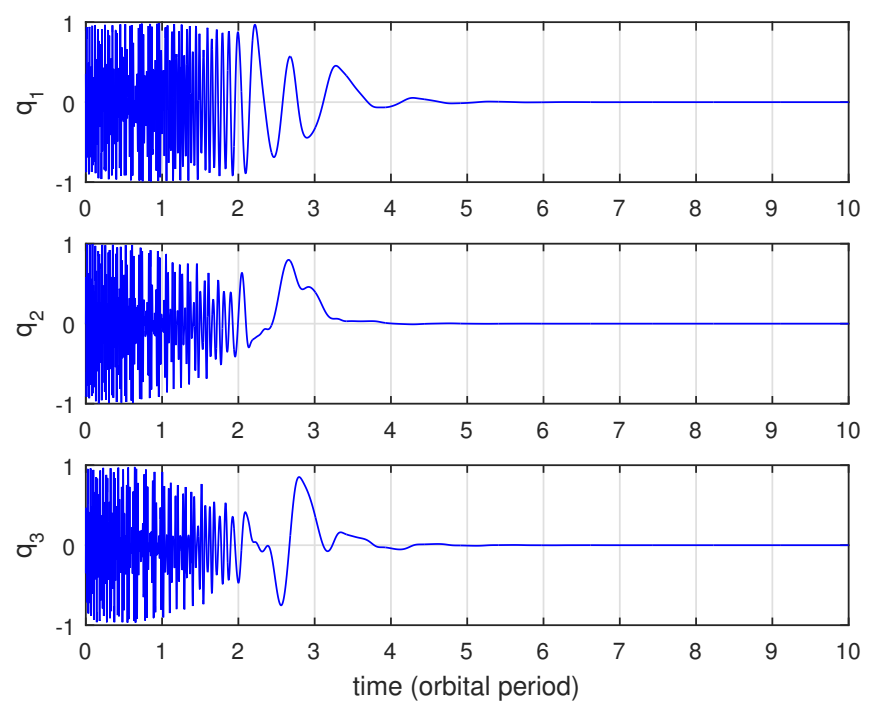

Fig. 4 Evolutions from initial state (23) with feedback gains (21).

\subsection{Variable Initial Conditions}

We now search for $\kappa_{p}$ and $\kappa_{d}$ using the robust optimization approach. We allow initial conditions to vary as follows: $0 \leq \rho \leq 1,0 \leq \phi \leq 2 \pi, 0 \leq \theta \leq \pi$, $\left|\omega_{01}\right| \leq 0.1,\left|\omega_{02}\right| \leq 0.1,\left|\omega_{03}\right| \leq 0.1,0 \leq \psi \leq 2 \pi$. Since the number of iterations in the internal loop critically determines the computational burden, we allowed a maximum of 2,200 function evaluations in each internal loop to solve the problem in reasonable times. The results of Procedure Solve minmax with maxeval_ext $=10,000$ and maxeval_int $=2,200$ are reported in the following Table 1.

The starting points used with SDBOX multistart are $\rho=0.5, \phi=\pi, \theta=\pi / 2$, $\psi=\pi$ and all the 8 combinations of extreme angular velocities

$$
\left[\begin{array}{lll}
0.1 & 0.1 & 0.1
\end{array}\right]^{\mathrm{T}},\left[\begin{array}{lll}
0.1 & 0.1 & -0.1
\end{array}\right]^{\mathrm{T}}, \ldots,\left[\begin{array}{lll}
-0.1 & -0.1 & -0.1
\end{array}\right]^{\mathrm{T}} \text {. }
$$


Table 1 Results of Solve min-max with maxiter_ext $=10,000$ and maxiter_int $=2,200$

\begin{tabular}{|c|c|c|c|}
\hline & $\begin{array}{c}\text { DIRECT } \\
+ \text { DIRECT }\end{array}$ & $\begin{array}{c}\text { DIRECT } \\
+ \text { SDBOX single start }\end{array}$ & $\begin{array}{c}\text { DIRECT } \\
+ \text { SDBOX multistart }\end{array}$ \\
\hline$\kappa_{p}$ & 205493.82716049382 & 203930.04115226338 & 209581.61865569273 \\
$\kappa_{d}$ & 117283950.61728397 & 154320987.65432101 & 117283950.61728397 \\
\hline$t_{s}$ & 3.359 & 3.142 & 3.742 \\
ext eval & 10,641 & 11,127 & 10,011 \\
\hline time (secs) & $172,242.6$ & $57,255.1$ & $89,640.8$ \\
\hline
\end{tabular}

From the algorithmic point of view, we observe that: $i$ ) in spite of the limited computations allowed in the internal loop, SDBOX multistart is able to reach the highest value of the objective $\left.t_{s} ; i i\right)$ DIRECT may perform a number of function evaluations slightly larger than the limit maxeval_ext, because it inevitably checks the termination condition only when the whole current set of candidate hyperrectangles has been examined (see Sect. 4.1); iii) the different results obtained in the solution of the lower-level problems determine a different evolution of the upper-level search. In order to determine which is the best choice among the 3 points above, we further extend the analysis in Table 2 . We fix parameters $\kappa_{p}$ and $\kappa_{d}$, and we allow many more function evaluations (eval) for solving the maximization problem, in order to increase accuracy. This is done by using: the global strategy; the local multistart strategy (which proved to overperform the single start one); and an exhaustive grid search (which is much slower and is used only as reference). 
Table 2 Thorough evaluation of the previously obtained gains.

\begin{tabular}{|c|c|c|c|}
\hline Gains & DIRECT & SDBOX multistart & Grid search \\
\hline$\kappa_{p}=205493.82716049382$ & 3.876 & 3.872 & 3.990 \\
$\kappa_{d}=117283950.61728397$ & & & \\
\hline$\kappa_{p}=203930.04115226338$ & 4.223 & 4.149 & 4.362 \\
$\kappa_{d}=154320987.65432101$ & & & \\
\hline$\kappa_{p}=209581.61865569273$ & 3.895 & 3.744 & 4.016 \\
$\kappa_{d}=117283950.61728397$ & & & \\
\hline
\end{tabular}

We obtain that the first point provides the smallest maximum settling time.

Thus, the robust optimal solution is given by:

$$
\kappa_{p}=205493.82716049382 \quad \kappa_{d}=117283950.61728397 .
$$

To evaluate the behavior of solution (24), we use it with the three different initial conditions reported in the previous subsection. If the initial state is given by (20), then we obtain $t_{s}=1.945$ (instead of 1.775$)$. Hence, there is a modest worsening. Indeed, no improvement was possible since gains (21) are optimal for state (20). The corresponding time evolutions are in Fig. 5.

If the initial state is given by (22), then we obtain $t_{s}=2.958$ (instead of 3.562). Hence, there is an improvement. This could be expected, though not guaranteed, since gains (21) have a higher worst-case result than gains (24). The corresponding time evolutions are represented in Fig. 6. 

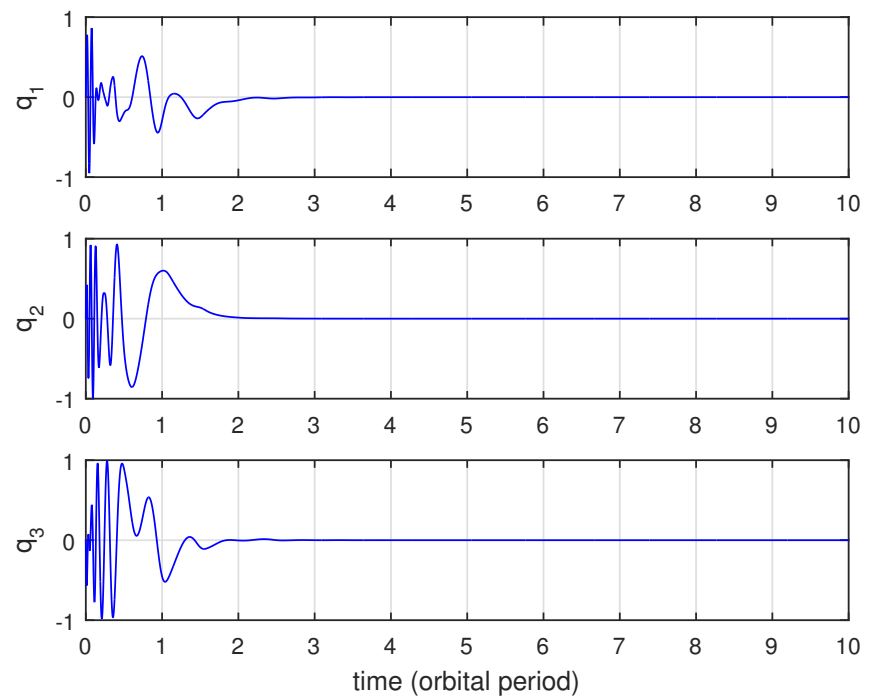

Fig. 5 Evolutions from initial state (20) with robust optimal feedback gains (24).
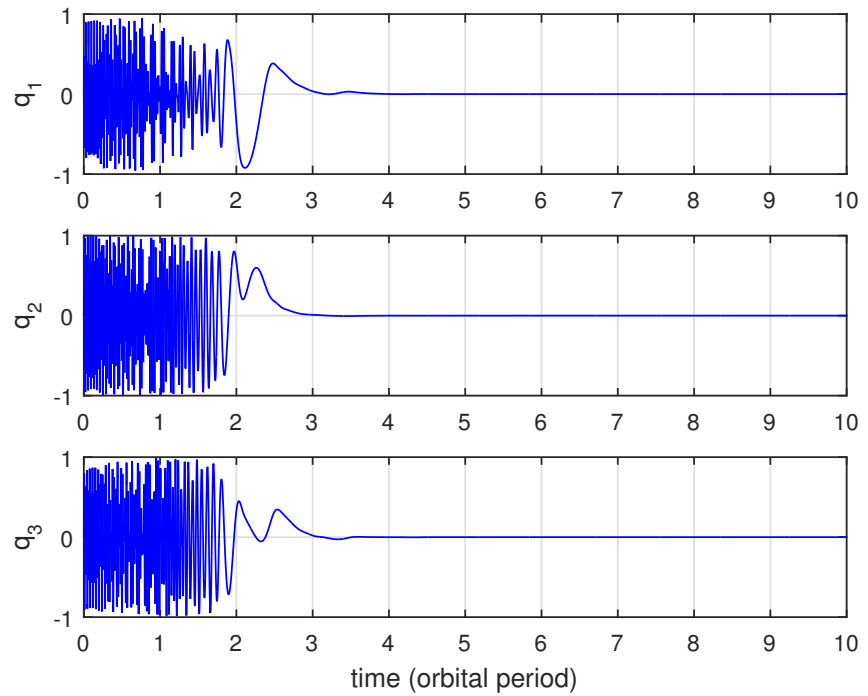

Fig. 6 Evolutions from initial state (22) with robust optimal feedback gains (24). 
Finally, if the initial state is given by $(23)$, then we obtain $t_{s}=3.370$ (instead of 4.338). Hence, there is a substantial improvement. Indeed, this was certain, since gains (24) have a worst-case result considerably better than gains (21). The corresponding time evolutions are represented in Fig. 7.
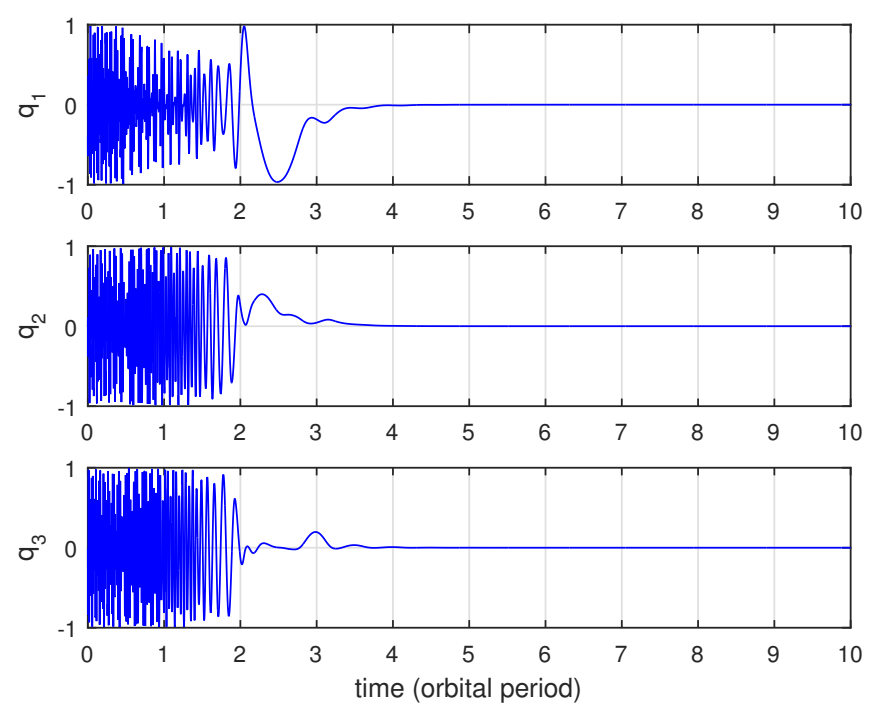

Fig. 7 Evolutions from initial state (23) with robust optimal feedback gains (24).

In conclusion, the gains computed with the proposed robust optimization approach offer improvements in the difficult situations. Moreover, even if they may be suboptimal in the easier cases, the balance appears beneficial.

\section{Conclusions}

PD-like feedback control can achieve attitude stabilization of a spacecraft using only magnetorquers. However, it needs a systematic method for determining 
the PD gains. Here, we formulate this problem as the selection of the gains that minimize the settling time under the worst initial conditions. This gives an upper bound on the value of the settling time obtained varying the initial conditions. This formulation turns out to be a very difficult min-max problem. Despite this, we propose a solution approach based on the use of derivative-free optimization algorithms, which is able to work without the need for analytically expressing the objective function, and which provides robust optimal solutions in reasonable times. It is shown through a case-study that the PD gains determined by the proposed method exhibit considerable advantages.

Acknowledgements The authors are grateful to Prof. Stefano Lucidi for several essential discussions and suggestions, and to Dr. Dennis G. Lucarelli for his help.

\section{References}

1. Sidi, M.J.: Spacecraft dynamics and control. Cambridge Univ. Press, New York (1997)

2. Silani, E., Lovera, M.: Magnetic spacecraft attitude control: A survey and some new results. Control Engineering Practice 13(3):357-371 (2005)

3. Lovera, M., Astolfi, A.: Spacecraft attitude control using magnetic actuators. Automatica 40(8):1405-1414 (2004)

4. Lovera, M., Astolfi, A.: Global magnetic attitude control of inertially pointing spacecraft. Journal of Guidance, Control, and Dynamics 28(5):1065-1067 (2005)

5. Celani, F.: Robust three-axis attitude stabilization for inertial pointing spacecraft using magnetorquers. Acta Astronautica 107:87-96 (2015)

6. Conn, A., Vicente, L.: Bilevel derivative-free optimization and its application to robust optimization. Optimization Methods Software 27, 561-577 (2012) 
7. Ciccazzo, A., Latorre, V., Liuzzi, G., Lucidi, S., Rinaldi, F.: Derivative-Free Robust Optimization for Circuit Design. Journal of Optimization Theory and Applications 164, $842-861(2015)$

8. Jones, D.R.: DIRECT global optimization. In: Floudas, C.A., Pardalos, P.M. (eds.) Encyclopedia of Optimization, pp. 725-735. Springer, Berlin (2009)

9. Jones, D.R., Perttunen, C.D., and Stuckman, B.E.: Lipschitzian optimization without the Lipschitz constant. J. of Optimization Theory and Application 79(1): 157-181 (1993)

10. Liuzzi, G., Lucidi, S., Piccialli, V.: A DIRECT-based approach exploiting local minimizations for the solution of large-scale global optimization problems. Computational Optimization and Applications 45, 353-375 (2010)

11. Lucidi, S., Sciandrone, M.: A Derivative-Free Algorithm for Bound Constrained Optimization. Computational Optimization and Applications 21, 119-142, 2002

12. Kolda, T.G., Lewis, R.M., Torczon, V.: Optimization by direct search: new perspectives on some classical and modern methods. SIAM Review 45(3), 385-482 (2003)

13. Wie, B.: Space vehicle dynamics and control. American institute of aeronautics and astronautics, Reston (VA) (2008)

14. Wertz, J.R. (ed.): Spacecraft attitude determination and control. Kluwer Academic Press, Dordrecht (1978)

15. Rodriguez-Vazquez, A.L., Martin-Prats, M.A., Bernelli-Zazzera, F.: Full magnetic satellite attitude control using ASRE method. In 1st IAA Conference on Dynamics and Control of Space Systems (2012)

16. Ogata, K.: Modern Control Engineering (4th ed.). PrenticeHall, Upper Saddle River (NJ) (2002)

17. Simon, E.: A perspective for optimization in systems and control: from LMIs to derivative-free methods. PhD thesis, Université Catholique de Louvain (2012)

18. Grippo, L., Lampariello, F., Lucidi, S.: Global convergence and stabilization of unconstrained minimization methods without derivatives. Journal of Optimization Theory and Applications 56(3), 385-406 (1988) 